\title{
Uma análise dos modelos de Thirlwall ou Solow para a economia brasileira
}

\author{
Luciano Nakabashi*
}

\begin{abstract}
RESUMO - Algumas evidências e teorias apontam para a existência de uma relação entre crescimento econômico e saldo da conta corrente. Ou seja, de acordo com essa abordagem é fundamental um bom desempenho do setor exportador de forma a manter uma conta corrente equilibrada para que um determinado país consiga manter um bom nível de investimentos e de crescimento de forma sustentável. Por outro lado, na literatura sobre crescimento existe uma ênfase na relação entre desempenho econômico e acumulação de fatores de produção. Levando em consideração essas duas abordagens teóricas, o presente estudo tem como objetivo estruturar, de forma inicial, um modelo teórico que relacione ambas e analisar o impacto da restrição externa nos investimentos em capital físico e capital humano e, consequentemente, no desempenho da economia brasileira, entre 1947 e 2008.
\end{abstract}

Palavras-chave: Exportações. Capital físico. Capital humano. Taxa de câmbio real. Desempenho econômico.

\section{INTRODUÇÃO}

Existe uma relação enfatizada por alguns economistas, há algum tempo, entre crescimento econômico e o saldo da conta de transações correntes, conhecido como lei de Thirlwall. Ou seja, de acordo com essa abordagem, é fundamental um bom desempenho do setor exportador de forma a manter uma conta corrente equilibrada para que um determinado país consiga manter elevados níveis de investimentos e de crescimento econômico.

O desempenho da balança comercial e da conta de transações correntes pode ser relevante no montante de investimentos e no crescimento de um determinado país pois, caso suceda uma piora nas mesmas, efeitos contracionistas ocorrem sobre os setores diretamente afetados pelo aumento das importações e/ou queda das exportações.

Outro ponto importante é que nenhum país pode crescer mais rápido que a taxa de crescimento com equilíbrio no BP, pelo menos no longo prazo, pois um déficit crescente na conta de transações correntes financiado pela conta capital aumentaria o risco de desvalorizações cambiais até o ponto em que não valesse mais a pena investir em tal região ou país. Desse modo, o país teria que se ajustar à nova situação através de medidas recessivas, sejam elas espontâneas ou forçadas. Finalmente, um déficit crescente na conta de transações correntes levaria o

\footnotetext{
*Doutor em economia pela Universidade Federal de Minas Gerais. É professor do Departamento de Economia da Universidade Federal do Paraná e pesquisador do CNPq. Endereço eletrônico: luciano.nakabashi@gmail.com.
} 
país ou região a praticar taxas de juros mais elevadas para atrair fluxos de capital, estimulando a valorização financeira do capital em prejuízo do investimento produtivo e do crescimento real.

Além de relaxar a restrição do setor externo, altas taxas de crescimento das exportações dinamizam a economia pela possibilidade que se abre na produção de bens mais intensivos em tecnologia, pois ocorre um processo de descolamento entre o consumo e a produção domésticos. Adicionalmente, os preços das exportações são relativamente inelásticos em relação à quantidade produzida, como argumentado por Eichengreen (2008). Vale mencionar ainda que um bom desempenho do setor exportador, com ganhos de participação deste no PIB, estimula o crescimento econômico porque ele possui maior capacidade de absorver tecnologia do resto do mundo e potencial de ganhos de learning by doing, quando comparado com os demais setores da economia (EICHENGREEN, 2008).

Por outro lado, como enfatizado por Solow (1956) ou Mankiw, Romer e Weil (1992), a única maneira de se alcançar crescimento econômico de forma sustentável é através da acumulação de fatores de produção, com especial ênfase ao progresso tecnológico. Ou seja, essa abordagem enfatiza o lado da oferta na determinação do crescimento econômico, enquanto aquela salienta fatores relacionados à demanda.

Considerando as diferentes abordagens acima citadas, no presente estudo buscamos estruturar um modelo formal que as relacione, assim como avaliarmos o impacto do desempenho do setor exportador e da conta de transações correntes nos investimentos em capital físico e capital humano e, consequentemente, no desempenho da economia brasileira, entre 1947 e 2008.

Além da presente introdução, realizamos a elaboração do modelo formal que serve de base para as estimações econométricas. Na terceira seção, a metodologia e os dados utilizados são mostrados, sendo apresentada a análise empírica em seguida.

\section{MODELO TEÓRICO}

Seguindo o modelo desenvolvido por Thirlwall e Hussain (1982), com a inclusão de fluxos de capitais, temos a seguinte equação de crescimento da renda com equilíbrio no balanço de pagamentos:

$$
y_{b, t}=\frac{(\theta \eta+\varphi+1)\left(p_{d, t}-p_{f, t}-e_{t}\right)+\theta \varepsilon z_{t}+(1-\theta)\left(f_{t}-p_{d, t}\right)}{\pi}
$$

Onde as letras minúsculas correspondem a taxas de crescimento das variáveis, $\mathrm{F}$ o valor nominal do fluxo de capitais em moeda doméstica, $\mathrm{P}_{d}$ é o preço das exportações, em moeda 
doméstica, $\mathrm{P}_{f}$ é o preço das importações, em moeda estrangeira, E é a taxa de câmbio nominal (preço doméstico da moeda estrangeira), $\varphi$ é a elasticidade-preço da demanda por importações $(\varphi<0), \pi$ é a elasticidade-renda da demanda por importações $(\pi>0), \eta$ é a elasticidade-preço da demanda por exportações $(\eta<0)$, Z é a renda mundial, $\varepsilon$ é a elasticidade-renda da demanda por exportações $(\varepsilon>0), \theta$ e $(1-\theta)$ são as parcelas das exportações e dos fluxos de capitais somados aos da conta de serviços no total das receitas obtidas pelo setor externo, ou seja, qual a parcela do total de importações é paga pelas exportações e qual é pela entrada de capitais, e o subscrito t representa o período em questão.

Nessa equação, a variável dependente $\left(\mathrm{y}_{b}\right)$ é a taxa de crescimento com equilíbrio no BP. Vale ressaltar que a equação (1) é sempre satisfeita no longo prazo, pois o país só pode crescer a taxas mais elevadas do que aquela que mantém a conta de transações correntes em equilíbrio caso ocorra uma entrada positiva de recursos externos via conta capital. No entanto, quando ocorrer uma reversão desses fluxos, o país terá que crescer a uma taxa inferior em relação àquela que equilibra o saldo das transações correntes ${ }^{1}$.

Considerando, nesse momento, o modelo de crescimento neoclássico, uma possível função de produção seria:

$$
Y_{t}=K_{t}^{\alpha} H_{t}^{\beta}(A L)_{t}^{1-\alpha-\beta}
$$

Em que Y é o nível de renda, K é o nível de capital físico, H é o nível de capital humano, A é o nível de tecnologia e L é a quantidade do fator trabalho usado no processo de produção. Novamente, o subscrito t se refere ao tempo t. Adicionalmente, $\alpha, \beta$, e $1-\alpha-\beta$ são as parcelas de cada um dos fatores na renda. Derivando a equação (2) em relação ao tempo e transformando as variáveis em taxa de crescimento, obtemos:

$$
y_{t}=\alpha k_{t}+\beta h_{t}+(1-\alpha-\beta) a_{t}+(1-\alpha-\beta) l_{t}
$$

Pela equação (3), notamos que o crescimento da renda só é possível caso ocorra uma elevação em pelo menos um dos fatores de produção da economia (capital físico, capital humano, trabalho ou tecnologia). Igualando as equações (1) e (3), chega-se a:

$$
\alpha k_{t}+\beta h_{t}+(1-\alpha-\beta) a_{t}+(1-\alpha-\beta) l_{t}=\frac{(\theta \eta+\varphi+1)\left(p_{d, t}-p_{f, t}-e_{t}\right)+\theta \varepsilon z_{t}+(1-\theta)\left(f_{t}-p_{d, t}\right)}{\pi}
$$

1 A equação (1) não precisa ser satisfeita no curto prazo, visto a possibilidade de ocorrência de variações no nível de reservas. 
Pela equação (4), notamos que a acumulação de fatores de uma determinada economia depende do resultado do BP da mesma. Notamos ainda que a conta externa é, de fato, uma restrição ao crescimento, pois este depende da acumulação dos fatores de produção.

Pela equação (4), podemos afirmar ainda que uma elevação do crescimento do resto do mundo, o que traz impactos positivos nas exportações, iria favorecer a acumulação de capital físico e humano, além de propiciar um crescimento do nível de tecnologia. Essa relação se deve a uma restrição externa menos rígida que, por sua vez, permite que ocorra acumulação dos fatores de produção com o consequente aumento da renda.

Isolando os fatores de produção capital físico e, posteriormente, capital humano do lado esquerdo da equação (4), encontramos:

$$
\begin{aligned}
& h_{t}=\frac{\theta \eta+\varphi+1}{\pi \beta}\left(p_{d, t}-p_{f, t}-e_{t}\right)+\frac{\theta \varepsilon}{\pi \beta} z_{t}+\frac{1-\theta}{\pi \beta}\left(f_{\mathrm{t}}-p_{d, t}\right)-\frac{\alpha}{\beta} h_{t}-\frac{1-\alpha-\beta}{\beta} l_{t}-\frac{1-\alpha-\beta}{\beta} a_{t} \\
& k_{t}=\frac{\theta \eta+\varphi+1}{\pi \alpha}\left(p_{d, t}-p_{f, t}-e_{t}\right)+\frac{\theta \varepsilon}{\pi \alpha} z_{t}+\frac{1-\theta}{\pi \alpha}\left(f_{\mathrm{t}}-p_{d, t}\right)-\frac{\beta}{\alpha} h_{t}-\frac{1-\alpha-\beta}{\alpha} l_{t}-\frac{1-\alpha-\beta}{\alpha} a_{t}
\end{aligned}
$$

As equações (5) e (6) formalizam a série de relações entre desempenho do setor externo, acumulação de fatores de produção e crescimento da renda destacada na seção anterior.

\section{METODOLOGIA E FONTE DOS DADOS}

A análise da primeira parte é feita a partir de dados anuais para o período que vai de 1950 até 2008. A escolha desse período se deve à limitação dos dados de capital físico para períodos anteriores.

Os dados do PIB da economia brasileira foram retirados do site do IPEA, que fornece o PIB a preços constantes e tem como fonte original o IBGE. A variável utilizada para mensurar a variação dos trabalhadores é a população residente em $1^{\circ}$ de julho, também do IBGE. Para mensurar o capital físico, foram utilizados os dados do estoque bruto total de capital fixo retirados do site do IPEA, sendo a fonte original Morandi e Reis (2004).

Para mensurar o capital humano, foi empregado o percentual de pessoas de 15 ou mais anos de idade que sabem ler e escrever um bilhete simples (IBGE), que é 1 (um) menos a taxa de analfabetismo. Como os dados estão disponíveis apenas a cada dez anos, essa porcentagem foi estimada, para os demais anos (entre os anos censitários), utilizando a função spline cúbica do software Matlab. O saldo da conta capital e financeira foi empregado para mensurar a entrada líquida de capitais e tem como fonte o Boletim do Banco Central do Brasil, assim como as 
exportações. Ambas as séries foram deflacionadas pelo Consumer Price Index (CPI) do US Bureau of Labor Statistics.

Para encontrar a taxa real de câmbio, além do CPI, fez-se o uso da taxa média de câmbio de venda - R \$/US $\$$ - comercial do boletim do Banco Central do Brasil e do IPC da Fipe e da FGV. O IPC dessas duas instituições foram somados e divididos por 2 (dois). Para mensurar o crescimento do resto do mundo, o PIB a preços constantes dos Estados Unidos foi utilizado como proxy. A fonte é o Bureau of Economic Accounts do US Department of Commerce. Todas as variáveis estão em valores reais.

A análise de regressão foi realizada com variáveis defasadas para controlar o problema de endogeneidade das variáveis explicativas. Em algumas especificações foram encontrados problemas de não normalidade dos resíduos. O método utilizado para corrigir tal problema foi o dos mínimos quadrados iterativos com redistribuição de pesos (Iteratively Rewveighted Least Squares - IRLS).

Outro problema grave encontrado em algumas especificações foi o de multicolinearidade. Para minimizar o problema, utilizamos a variável exportações no lugar do PIB dos EUA. Em alguns momentos, essa substituição foi suficiente para eliminar o problema, enquanto que em outros a correlação entre algumas variáveis explicativas continuou elevada, o que torna os resultados encontrados nessas especificações menos confiáveis.

\section{RELAÇÕES ENTRE RENDA, FATORES DE PRODUÇÃO E RESTRIÇÃO EX- TERNA}

$\mathrm{Na}$ Tabela 1 são apresentados os resultados que testam a importância da restrição externa no processo de acumulação de capital físico, de acordo com a especificação apresentada na equação (5). Os resultados apresentados nas quatro primeiras colunas mostram que cada uma das variáveis que relaxa a restrição ao crescimento é relevante para explicar as variações no estoque de capital físico da economia brasileira quando consideradas isoladamente.

Quando controlamos para os efeitos de todas as variáveis que relaxam a restrição externa (colunas 5 e 6), os resultados mostram que as variáveis fundamentais são crescimento do resto do mundo e exportações. Quando as quatro variáveis são consideradas como explicativas ao mesmo tempo (coluna 7), os resultados apontam que a variável relevante para explicar o relaxamento da restrição externa é o crescimento do resto do mundo.

Incluindo a variável capital humano de forma a aproximar da especificação apresentada na equação (5), verificamos a perda de significância da variável renda do resto do mundo na coluna (8). No entanto, o FIV indica que a inclusão daquela introduziu o problema da mul- 
ticolinearidade, o que infla a variância e faz com que os coeficientes percam sua significância. Ao substituir a variável renda do resto do mundo pelas exportações, verificamos que não há problemas de multicolinearidade e estas passam a ser significativas ao nível de $1 \%$ na explicação da acumulação de capital físico.

TABELA 1 - REGRESSÕES TESTANDO A RESTRIÇÃO EXTERNA NA ACUMULAÇÃO DE CAPITAL FÍSICO DE ACORDO COM A ESPECIFICAÇÃO APRESENTADA NA EQUAÇÃO (5)

\begin{tabular}{|c|c|c|c|c|c|c|c|c|c|c|c|}
\hline \multirow{3}{*}{ Variáveis } & \multicolumn{11}{|c|}{ Variável dependente: ln do capital físico } \\
\hline & (1) & $(2)$ & (3) & (4) & (5) & (6) & (7) & (8) & (9) & (10) & (11) \\
\hline & MQO & MQO & IRLS & MQO & MQO & IRLS & MQO & MQO & IRLS & MQO & IRLS \\
\hline \multirow[t]{2}{*}{$\operatorname{lne}(\mathrm{n}-1)$} & 1.289 & & & & -0.278 & -0.059 & -0.269 & -0.229 & -0.224 & -0.0180 & -0.240 \\
\hline & $(0.321)^{*}$ & & & & $(0.083)^{*}$ & $(0.115)$ & $(0.084)^{*}$ & $(0.051)^{*}$ & $(0.031)^{*}$ & $(0.038)$ & $(0.031)^{*}$ \\
\hline \multirow[t]{2}{*}{$\ln y^{*}(n-1)$} & & 1.829 & & & 1.913 & & 1.792 & 0.083 & & -1.816 & \\
\hline & & $(0.051)^{*}$ & & & $(0.062)^{*}$ & & $(0.144)^{*}$ & $(0.192)$ & & $(0.229)^{*}$ & \\
\hline \multirow[t]{2}{*}{$\ln x(n-1)$} & & & 1.029 & & & 1.022 & 0.082 & & 0.213 & & 0.190 \\
\hline & & & $(0.048)^{*}$ & & & $(0.056)^{*}$ & $(0.089)$ & & $(0.028)^{*}$ & & $(0.038)^{*}$ \\
\hline \multirow[t]{2}{*}{$\ln f(n-1)$} & & & & 0.957 & 0.072 & -0.014 & 0.062 & 0.021 & 0.046 & 0.034 & 0.054 \\
\hline & & & & $(0.278)^{*}$ & $(0.063)$ & $(0.094)$ & $(0.064)$ & $(0.038)$ & $(0.024)^{* * *}$ & $(0.023)$ & $(0.024)^{* *}$ \\
\hline \multirow[t]{2}{*}{$\operatorname{lnh}(n-1)$} & & & & & & & & 6.192 & 5.451 & -1.470 & 4.760 \\
\hline & & & & & & & & $(0.638)^{*}$ & $(0.151)^{*}$ & $(0.884)^{* * *}$ & $(0.911)^{*}$ \\
\hline \multirow[t]{2}{*}{$\ln l(n-1)$} & & & & & & & & & & 5.838 & 0.333 \\
\hline & & & & & & & & & & $(0.606)^{*}$ & $(0.430)$ \\
\hline \multirow[t]{2}{*}{ C } & 6.907 & -8.384 & -3.262 & -2.524 & -9.758 & -2.938 & -9.467 & -19.918 & -18.499 & -79.138 & -21.561 \\
\hline & $(0.163)^{*}$ & $(0.440)^{*}$ & $(0.497)^{*}$ & $(2.869)$ & $(0.628)^{*}$ & $(0.867)^{*}$ & $(0.702)^{*}$ & $(1.114)^{*}$ & $(0.431)$ & $(6.182)^{*}$ & $(3.940)^{*}$ \\
\hline $\mathrm{N}$ & 58 & 58 & 58 & 58 & 58 & 58 & 58 & 58 & 58 & 58 & 58 \\
\hline $\mathrm{R}^{2}$ & 0.2240 & 0.9582 & 0.872 & 0.1747 & 0.9672 & 0.8740 & 0.9678 & 0.9882 & 0.9921 & 0.9958 & 0.9922 \\
\hline $\mathrm{R}^{2}$ ajust. & 0.2102 & 0.9574 & 0.870 & 0.1600 & 0.9654 & 0.8670 & 0.9653 & 0.9873 & 0.9915 & 0.9954 & 0.9914 \\
\hline $\mathrm{F}$ & 16.17 & 1283.48 & 462.61 & 11.86 & 531.43 & 178.28 & 397.72 & 1109.21 & 2881.55 & 2443.88 & 2394.71 \\
\hline Prob $>$ F & 0.0002 & 0.0000 & 0.0000 & 0.0011 & 0.0000 & 0.0000 & 0.0000 & 0.0000 & 0.0000 & 0.0000 & 0.0000 \\
\hline \multirow[t]{2}{*}{ FIV (1) } & & & & & 1.79 & 1.65 & 9.81 & 47.53 & 6.75 & 635.29 & 313.14 \\
\hline & & & & & $\ln y^{*}(n-1)$ & $\ln x(n-1)$ & $\ln y^{*}(n-1)$ & $\ln y^{*}(n-1)$ & $\operatorname{lnh}(n-1)$ & $\ln 1(n-1)$ & $\ln l(n-1)$ \\
\hline \multirow[t]{2}{*}{ FIV (2) } & & & & & 1.53 & 1.36 & 9.06 & 46.65 & 6.35 & 244.52 & 253.97 \\
\hline & & & & & $\ln e(n-1)$ & $\ln (\mathrm{n}-1)$ & $\ln x(n-1)$ & $\operatorname{lnh}(n-1)$ & $\ln x(n-1)$ & $\operatorname{lnh}(n-1)$ & $\operatorname{lnh}(n-1)$ \\
\hline \multirow[t]{2}{*}{ FIV (3) } & & & & & 1.23 & 1.27 & 1.55 & 1.55 & 1.48 & 184.31 & 12.13 \\
\hline & & & & & $\ln f(n-1)$ & $\ln f(n-1)$ & $\ln e(n-1)$ & $\ln e(n-1)$ & $\ln e(n-1)$ & $\ln y^{*}(\mathrm{n}-1)$ & $\ln x(n-1)$ \\
\hline Ass $/$ Cur $\chi^{2}$ & 3.26 & 5.70 & 11.43 & 1.23 & 1.60 & 10.02 & 0.71 & 4.10 & 8.62 & 3.37 & 8.47 \\
\hline Prob $>\chi^{2}$ & 0.1959 & 0.0579 & 0.0033 & 0.5399 & 0.4490 & 0.0067 & 0.7008 & 0.1284 & 0.0134 & 0.1853 & $0.0145^{*}$ \\
\hline Traço & 0 & 0 & 0 & 0 & 1 & 2 & 3 & 2 & 3 & 3 & 4 \\
\hline Autovalor & 0 & 0 & 0 & 1 & 1 & 0 & 1 & 2 & 2 & 3 & 4 \\
\hline CIS & 2 & 3 & 2 & 2 & 3 & 2 & 1 & 4 & 4 & 4 & 4 \\
\hline
\end{tabular}

NOTAS: $* * *$ significativo a $10 \%, * *$ significativo $5 \%$, * significativo a $1 \%$. Ass /Cur $\chi^{2}$ fornece o valor do $\chi^{2}$ para o teste de assimetria e curtose para normalidade dos resíduos da equação original, ou seja, sem correção para o problema. Aquelas regressões que apresentaram problema de não normalidade dos resíduos ao nível de $5 \%$ estimadas de forma a corrigir o problema. A variável explicada é o logaritmo natural do estoque real de capital físico da economia brasileira. Todas as variáveis empregadas como explicativas estão em termos reais, em logaritmo natural (ln) e estão defasadas em um período (n-1), sendo elas: a taxa real de câmbio [lne(n-1)]; o nível do PIB dos EUA [lny*(n-1)]; o nível das exportações [lnx(n-1)]; o nível do fluxo líquido de capitais [lnf(n-1)]; o estoque de capital humano [ $\operatorname{lnh}(\mathrm{n}-1)]$; e a população residente [nll(n-1)]. C é o intercepto e $\mathrm{N}$ é o número de observações. F é o valor do teste F para testar a significância global do modelo, Prob > F é o seu p-valor, FIV é o Fator de Inflação da Variância, com as variáveis relacionadas ao seu valor logo abaixo, Ass/Cur $\chi^{2}$, como já dito, é o valor do teste $\chi^{2}$ para testar a normalidade dos resíduos e Prob $>\chi^{2}$ é o seu p-valor. O traço e autovalor são testes para verificar a quantidade de vetores de cointegração entre as variáveis, com as quantidades apresentadas na mesma linha, de acordo com cada teste. O CIS é o Critério de Informação de Schwarz para determinar se deve constar no teste de Cointegração de Johansen a 
ausência de intercepto, de tendência e de tendência determinística (1), intercepto, ausência de tendência na Equação de Cointegração (EC) e de tendência determinística (2), tendência determinística, intercepto e ausência de tendência na EC (3), tendência determinística, intercepto e tendência na EC (4), tendência determinística quadrática, intercepto e tendência na EC (5).

Com a especificação da equação (5) completa, verificamos que os resultados são semelhantes. A diferença é que a influência da renda do resto do mundo passa a ser negativa e seu coeficiente estatisticamente diferente de zero, ao nível de significância de 1\%. No entanto, precisamos ter cuidado ao interpretar os resultados pelo grave problema de multicolinearidade apresentado.

O problema da multicolinearidade quando se emprega o nível de exportações ainda é pequeno com a especificação completa, de acordo com os resultados apresentados na última coluna. Seu efeito no estoque do capital físico continua positivo e significativo, ao nível de 1\%, indicando a importância das exportações para relaxar a restrição externa e estimular a acumulação desse fator de produção.

Como podemos verificar nas últimas colunas da Tabela 1, a existência de vetores de cointegração quando utilizamos todas as variáveis que relaxam a restrição externa (colunas 5, 6 e 7) confirmam que a relação entre elas não é espúria, ou seja, a restrição externa é, realmente, relevante na explicação da acumulação do capital físico. Os modelos mais completos, com seus resultados apresentados nas últimas quatro colunas, também apresentam pelo menos um vetor de cointegração, indicando a relevância da relação entre as variáveis.

Na Tabela 2 estão os resultados da especificação apresentada na equação (6). Nela, a variável explicada é o logaritmo natural do capital humano. As 11 especificações apresentadas são análogas àquelas da Tabela 1 . Nas quatro primeiras colunas estão os efeitos de cada uma das variáveis que relaxa a restrição externa sobre o fator capital humano. Os resultados indicam que cada uma destas têm um efeito positivo e significativo na acumulação do fator capital humano, quando consideradas isoladamente.

Quando introduzimos as quatro variáveis em uma mesma equação (coluna 7), notamos que o efeito da renda continua positivo e significativo, enquanto o coeficiente das exportações se torna negativo e significativo, o que indica que a variável mais adequada no modelo é a renda do resto do mundo. Quando inserimos o capital físico na análise, o coeficiente da variável renda do resto do mundo continua positivo e significativo, apesar do problema da multicolinearidade, como apresentado na coluna (8). Ao utilizar as exportações no lugar da renda do resto do mundo, os resultados mudam de forma considerável, conforme exposto na coluna (9). Neste caso, o coeficiente das exportações passa a ter um sinal negativo e significativo, enquanto a depreciação do câmbio passa a exercer um efeito positivo e significativo na variação do capital humano. 
Introduzindo a variável proxy para o fator trabalho, os resultados apresentados nas duas últimas colunas da Tabela 2 nos mostram que a depreciação do câmbio tem um papel relevante na acumulação do capital humano. Adicionalmente, essa variável não apresenta problemas de multicolinearidade com as demais. O coeficiente da variável proxy para crescimento do resto do mundo apresenta um sinal negativo e é estatisticamente diferente de zero, ao nível de $1 \%$. No entanto, o elevado grau de multicolinearidade entre as variáveis compromete a estimação dos mesmos.

TABELA 2 - REGRESSÕES TESTANDO A RESTRIÇÃO EXTERNA NA ACUMULAÇÃO DE CAPITAL HUMANO DE ACORDO COM A ESPECIFICAÇÃO APRESENTADA NA EQUAÇÃO (6)

\begin{tabular}{|c|c|c|c|c|c|c|c|c|c|c|c|}
\hline \multirow{3}{*}{ Variáveis } & \multicolumn{11}{|c|}{ Variável dependente: ln do capital humano } \\
\hline & (1) & (2) & (3) & (4) & (5) & $(6)$ & $(7)$ & $(8)$ & (9) & (10) & (11) \\
\hline & MQO & IRLS & IRLS & MQO & IRLS & IRLS & MQO & IRLS & IRLS & IRLS & MQO \\
\hline \multirow[t]{2}{*}{$\ln e(n-1)$} & 0.226 & & & & -0.014 & 0.032 & -0.011 & 0.010 & 0.0431 & 0.013 & 0.017 \\
\hline & $(0.048)^{*}$ & & & & $(0.007)^{* * *}$ & $(0.021)$ & $(0.010)$ & $(0.004)^{* *}$ & $(0.005)^{*}$ & $(0.004)^{*}$ & $(0.004)^{*}$ \\
\hline \multirow[t]{2}{*}{$\ln y^{*}(n-1)$} & & 0.271 & & & 0.275 & & 0.321 & 0.135 & & -0.122 & \\
\hline & & $(0.004)^{*}$ & & & $(0.0065)^{*}$ & & $(0.017)^{*}$ & $(0.011)^{*}$ & & $(0.034)^{*}$ & \\
\hline \multirow[t]{2}{*}{$\ln x(n-1)$} & & & 0.153 & & & 0.149 & -0.023 & & -0.022 & & -0.022 \\
\hline & & & $(0.008)^{*}$ & & & $(0.010)^{*}$ & $(0.010)^{* *}$ & & $(0.005)^{*}$ & & $(0.004)^{*}$ \\
\hline \multirow[t]{2}{*}{$\ln f(n-1)$} & & & & 0.144 & 0.011 & 0.003 & 0.012 & 0.004 & 0.001 & 0.004 & 0.007 \\
\hline & & & & $(0.043)^{*}$ & $(0.005)^{* *}$ & $(0.017)$ & $(0.007)^{* * *}$ & $(0.003)^{* * *}$ & $(0.003)$ & $(0.003)$ & $(0.003)^{* *}$ \\
\hline \multirow[t]{2}{*}{$\operatorname{lnk}(n-1)$} & & & & & & & & 0.071 & 0.162 & -0.023 & 0.047 \\
\hline & & & & & & & & $(0.006)^{*}$ & $(0.004)^{*}$ & $(0.017)$ & $(0.012)^{*}$ \\
\hline \multirow[t]{2}{*}{$\operatorname{lnl}(n-1)$} & & & & & & & & & & 0.632 & 0.317 \\
\hline & & & & & & & & & & $(0.088)^{*}$ & $(0.033)^{*}$ \\
\hline \multirow[t]{2}{*}{ C } & 4.197 & 1.952 & 2.708 & 2.793 & 1.810 & 2.702 & 1.630 & 2.547 & 3.310 & -6.246 & -1.778 \\
\hline & $(0.024)^{*}$ & $(0.038)^{*}$ & $(0.078)^{*}$ & $(0.448)^{*}$ & $(0.054)^{*}$ & $(0.159)^{*}$ & $(0.082)^{*}$ & $(0.063)^{*}$ & $(0.041)^{*}$ & $(1.241)^{*}$ & $(0.528)^{*}$ \\
\hline $\mathrm{N}$ & 58 & 58 & 58 & 58 & 58 & 58 & 58 & 58 & 58 & 58 & 58 \\
\hline $\mathrm{R}$ & 0.2876 & 0.9788 & 0.8443 & $0.1 c$ & 0.9794 & 0.8567 & 0.9815 & 0.9937 & 0.9922 & 0.9965 & 0.9972 \\
\hline $\mathrm{R}^{2}$ ajust. & 0.2748 & 0.9784 & 0.8416 & 0.1487 & 0.9783 & 0.8487 & 0.9801 & 0.9932 & 0.9916 & 0.9962 & 0.9969 \\
\hline $\mathrm{F}$ & 22.60 & 3781.37 & 415.00 & 10.96 & 1605.40 & 129.50 & 703.95 & 5243.38 & 2780.56 & 4282.36 & 3642.85 \\
\hline Prob $>$ F & 0.0000 & 0.0000 & 0.0000 & 0.0016 & 0.0000 & 0.0000 & 0.0000 & 0.0000 & 0.0000 & 0.0000 & 0.0000 \\
\hline \multirow[t]{2}{*}{ FIV (1) } & & & & & 1.79 & 1.65 & 9.81 & 35.63 & 8.07 & 1011.06 & 117.82 \\
\hline & & & & & $\ln y^{*}(n-1)$ & $\ln x(n-1)$ & $\ln y^{*}(\mathrm{n}-1)$ & $\ln y^{*}(\mathrm{n}-1)$ & $\ln x(n-1)$ & $\operatorname{lnl}(n-1)$ & $\operatorname{lnl}(n-1)$ \\
\hline \multirow[t]{2}{*}{ FIV (2) } & & & & & 1.53 & 1.36 & 9.06 & 32.13 & 7.87 & 309.98 & 111.56 \\
\hline & & & & & $\ln e(n-1)$ & $\ln e(n-1)$ & $\ln x(n-1)$ & $\operatorname{lnk}(\mathrm{n}-1)$ & $\operatorname{lnk}(\mathrm{n}-1)$ & $\ln y^{*}(\mathrm{n}-1)$ & $\operatorname{lnk}(\mathrm{n}-1)$ \\
\hline \multirow[t]{2}{*}{ FIV (3) } & & & & & 1.23 & 1.27 & 1.55 & 1.85 & 1.37 & 268.04 & 8.18 \\
\hline & & & & & $\ln f(n-1)$ & $\ln f(n-1)$ & $\ln (n-1)$ & $\operatorname{lne}(n-1)$ & $\ln e(n-1)$ & $\ln k(n-1)$ & $\ln x(n-1)$ \\
\hline Ass $/$ Cur $\chi^{2}$ & 1.32 & 8.37 & 12.81 & 0.44 & 11.45 & 10.06 & 3.12 & 14.44 & 11.03 & 15.83 & 3.40 \\
\hline Prob $>\chi^{2}$ & 0.5179 & 0.0152 & 0.0017 & 0.8014 & 0.0033 & 0.0065 & 0.2105 & 0.0007 & 0.0040 & 0.0004 & 0.1826 \\
\hline Traço & 1 & 1 & 0 & 1 & 1 & 2 & 1 & 2 & 3 & 3 & 4 \\
\hline Autovalor & 1 & 1 & 0 & 1 & 1 & 2 & 1 & 2 & 2 & 3 & 4 \\
\hline CIS & 1 & 4 & 3 & 1 & 4 & 1 & 4 & 4 & 4 & 4 & 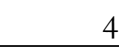 \\
\hline
\end{tabular}

NOTAS: $* * *$ significativo a $10 \%,{ }^{* *}$ significativo $5 \%, *$ significativo a $1 \%$. Ass /Cur $\chi^{2}$ fornece o valor do $\chi^{2}$ para o teste de assimetria e curtose para normalidade dos resíduos da equação original, ou seja, sem correção para o problema. Aquelas regressões que apresentaram problema de não normalidade dos resíduos ao nível de $5 \%$ estimadas de forma a corrigir o problema. A variável explicada é o logaritmo natural do estoque de capital humano da economia brasileira. Todas as variáveis empregadas como explicativas estão em termos reais, em logaritmo natural (ln) e estão defasadas em um período (n-1), sendo elas: a taxa real de câmbio [lne(n-1)]; o nível do PIB dos EUA [lny*(n-1)]; o nível das exportações [lnx(n-1)]; o nível do fluxo líquido de capitais [lnf(n-1)]; o estoque de capital físico [ $\operatorname{lnk}(\mathrm{n}-1)]$; e a população residente [lnl(n-1)]. C é o intercepto e N é o número de observações. F é o valor do teste F para testar a significância global do modelo, Prob > F é o seu p-valor, FIV é o 
Fator de Inflação da Variância, com as variáveis relacionadas ao seu valor logo abaixo, Ass/Cur $\chi^{2}$, como já dito, é o valor do teste $\chi^{2}$ para testar a normalidade dos resíduos e Prob $>\chi^{2}$ é o seu p-valor. O traço e autovalor são testes para verificar a quantidade de vetores de cointegração entre as variáveis, com as quantidades apresentadas na mesma linha de acordo com cada teste. O CIS é o Critério de Informação de Schwarz para determinar se deve constar no teste de Cointegração de Johansen a ausência de intercepto, de tendência e de tendência determinística (1), intercepto, ausência de tendência na Equação de Cointegração (EC) e de tendência determinística (2), tendência determinística, intercepto e ausência de tendência na EC (3), tendência determinística, intercepto e tendência na EC (4), tendência determinística quadrática, intercepto e tendência na EC (5).

Nas últimas colunas da Tabela 2, verificamos que praticamente todas as diferentes especificações relacionando as variáveis explicativas e a explicada possuem pelo menos um vetor de cointegração, sugerindo que a relação entre as variáveis que relaxam a restrição externa realmente importa na determinação do nível de capital humano na economia brasileira.

Pelo elevado grau de multicolinearidade quando se introduz a proxy para o fator trabalho, seria prudente não considerar os resultados das duas últimas regressões na análise dos resultados, sendo o mesmo válido para as duas últimas especificações apresentadas na Tabela 1. Excluindo esses resultados, as estimações apresentadas acima indicam que variações da renda mundial, através de seus efeitos sobre as exportações da economia, são relevantes na acumulação dos fatores de produção capital físico e capital humano.

O efeito da depreciação cambial parece ser negativo na acumulação de capital físico quando se controla para as demais variáveis. No entanto, não podemos esquecer que o câmbio tem impactos positivos sobre as exportações que, por sua vez, influenciam positivamente o nível desse fator de produção. Seus efeitos negativos podem ser porque, uma vez controlado seus impactos sobre as exportações, depreciações cambiais tornam os bens de capital importados mais caros.

A relação das depreciações cambiais com variações no nível de capital humano é ambígua. Em algumas estimações seus efeitos se mostram negativos (coluna 5), enquanto em outras eles são positivos (colunas 8 e 9). De qualquer forma, é difícil imaginar que o efeito das depreciações cambiais seja positivo na acumulação dos fatores de produção, fora seus estímulos sobre as exportações. Uma possibilidade seria o favorecimento dos setores que concorrem com os importados, relaxando ainda mais a restrição externa.

\section{CONCLUSÃO}

Existe uma grande discussão sobre os principais fatores que determinam o crescimento de uma economia. No entanto, ela é feita de uma forma separada, enfatizando diferentes elementos: alguns que destacam fatores de oferta e outros de demanda.

No presente estudo foi feito um esforço no sentido de elaborar um modelo ainda em 
fase inicial, de modo a explorar as inter-relações existentes entre essas distintas abordagens, para avançar no sentido da compreensão dos fatores-chave que determinam o crescimento da economia brasileira.

Os resultados encontrados mostram que a restrição externa é um elemento importante para explicar a acumulação de fatores de produção da economia. Nesse sentido, podemos pensar no modelo de Thirlwall como sendo de restrição ao crescimento e não de crescimento propriamente dito, pois uma economia que não encontre problemas no Balanço de Pagamentos não necessariamente alcançará um bom desempenho caso outros elementos limitem a acumulação dos fatores de produção.

\section{REFERÊNCIAS}

EICHENGREEN, B. The real exchange rate and economic growth. Comission on Growth and Development, Working Paper, n. 4, 2008.

MANKIW, N. G.; ROMER, D.; WEIL, D. A contribution to the empirics of economic growth. The Quarterly Journal of Economics, v. 107, n. 2, p. 407- 437, 1992.

SOLOW, R. M. A contribution to the theory of economic growth. The Quarterly Journal of Economics, v. 70, n. 1, p. 65-94, 1956.

THIRLWALL, A. P. The balance of payments constraint as a explanation of international growth rate differences. Banca Nazionale del Lavoro Quarterly Review, v. 128, n. 791, p. 450-453, 1979.

THIRLWALL, A. P.; HUSSAIN, M. N. The balance of payments constraint, capital flows and growth rate differences between developing countries. Oxford Economic Papers, v. 34, n. 3, p. 498-510, 1982. 